\title{
Estado nutricional de crianças com paralisia cerebral
}

\author{
Nutritional status of children \\ with cerebral palsy
}

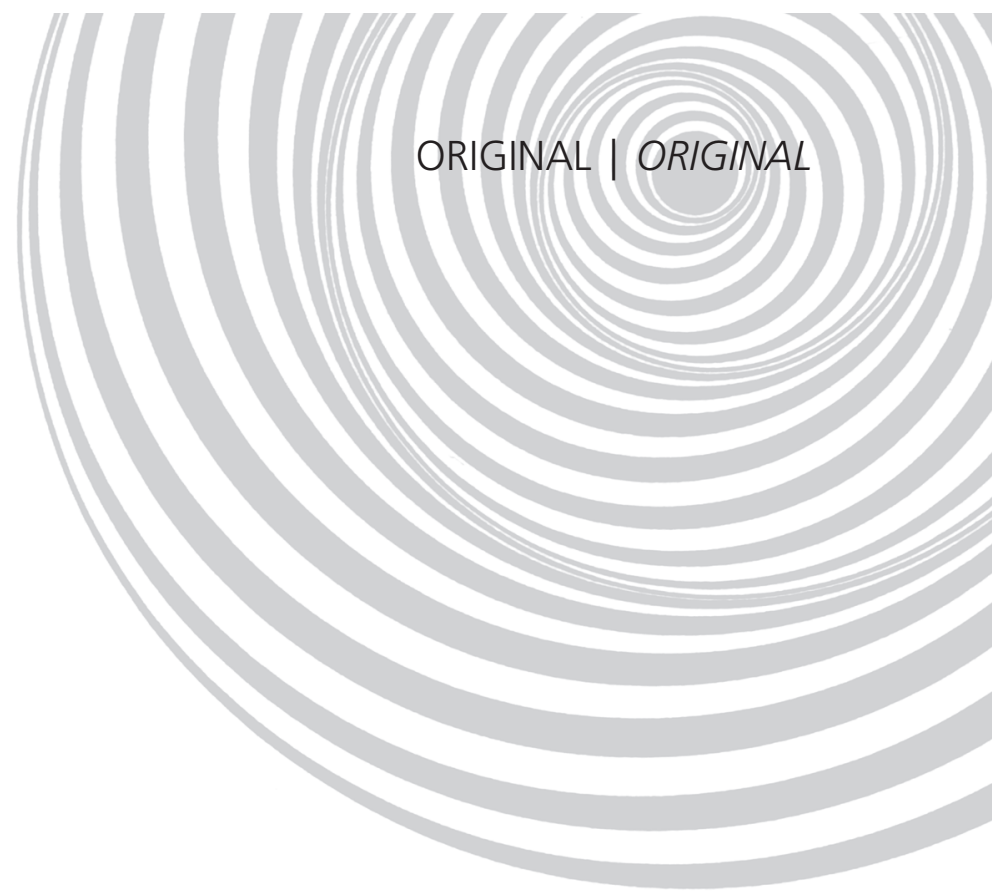

Ana Lúcia Alves CARAM ${ }^{1}$

André Moreno MORCILLO ${ }^{1}$

Elizete Aparecida Lomazi da Costa PINTO'

R E S U M O

Objetivo

Avaliar o estado nutricional de 114 crianças com paralisia cerebral, entre 2 e 12,9 anos de idade.

Métodos

Estudo transversal incluindo crianças atendidas nos ambulatórios de gastropediatria, ortopedia e neuropediatria de um hospital terciário universitário. Os indicadores nutricionais antropométricos estudados foram: peso, altura, índice de massa corporal, área muscular e de gordura do braço.

\section{Resultados}

A frequência de comprometimento nutricional (Escore-Z $\leq-2)$, de acordo com os indicadores antropométricos para a idade e a média com o desvio-padrão, foram: 50,9\% para peso, com média de $-2,19$ ( $D P=2,48), 38,6 \%$ para altura, média: $-1,66(\mathrm{DP}=1,50)$, 45,6\% para índice de massa corporal, média: $-1,74$ ( $\mathrm{DP}=2,75), 29,8 \%$ para área muscular do braço, média: -1,03 $(\mathrm{DP}=1,73)$ e 35,1\% para área de gordura do braço, média: $-1,15$ $(\mathrm{DP}=1,41)$.

\section{Conclusão}

A desnutrição foi frequente em crianças com paralisia cerebral. As crianças com paralisia cerebral do tipo tetraparético apresentaram os indicadores antropométricos mais comprometidos: peso e índice de massa corporal, com $72,4 \%$ e $73,1 \%$ das crianças com Escore- $Z \leq-2$ respectivamente.

Termos de indexação: Avaliação nutricional. Crescimento. Desnutrição. Paralisia cerebral.

A B S T R A C T

\section{Objective}

The aim of this study was to evaluate the nutritional status of 114 children aged 2 to 12.9 years with cerebral palsy.

\footnotetext{
1 Universidade Estadual de Campinas, Faculdade de Ciências Médicas, Departamento de Pediatria. Caixa Postal 6111, Cidade Universitária, 13083-970, Campinas, SP, Brasil. Correspondência para/Correspondence to: A.L.A. CARAM. E-mail: <anacaram@fcm.unicamp.br>.
} 
212 | A.L.A. CARAM et al.

\section{Methods}

This cross-sectional study included children treated at the gastropediatric, orthopedic and neuropediatric outpatient clinics of a tertiary university hospital. The studied anthropometric indicators were: weight, height, body mass index and mid-upper arm fat and muscle area.

\section{Results}

The rate of malnutrition (Z-score $\leq-2$ ) according to the anthropometric indicators for age, mean and standard deviation (SD) were: $50.9 \%$ for weight, with a mean of -2.19 (SD=2.48), 38.6\% for height, mean: -1.66 $(S D=1.50), 45.6 \%$ for body mass index, mean: $-1.74(S D=2.75), 29.8 \%$ for mid-upper arm muscle area, mean: $-1.03(S D=1.73)$ and $35.1 \%$ for mid-upper arm fat area, mean: $-1.15(S D=1.41)$.

\section{Conclusion}

Malnutrition was common in children with cerebral palsy. The anthropometric indicators were worse in children with tetraparetic cerebral palsy: $72.4 \%$ and $73.1 \%$ of the children had Z-scores $\leq-2$ for weight and body mass index, respectively.

Indexing terms: Nutritional assessment. Growth. Malnutrition. Cerebral palsy.

\section{N T R O D U Ç Ã O}

As bases da atenção à saúde das crianças incluem a avaliação do estado nutricional e a identificação precoce de fatores de risco para desenvolvimento de deficiências nutricionais".

Portadores de Paralisia Cerebral (PC) são, reconhecidamente, pacientes com risco para comprometimento nutricional. Relatos na literatura internacional identificaram altas frequências de desnutrição, e os distúrbios da deglutição são apontados como um fator de risco associado ao agravo nutricional ${ }^{2-7}$.

Avaliar o crescimento de crianças com paralisia cerebral é um desafio determinado por fatores como a incapacidade para assumir posição ortostática, restrições de movimento articular, desvios da coluna vertebral, contraturas musculares e baixo nível de cooperação. Ultrapassadas essas dificuldades técnicas, o profissional depara-se com o fato de que referências antropométricas para essa população são escassas e restritas².

A ocorrência estimada de pacientes com PC pode chegar a 7 crianças para cada mil nascidos vivos, em países subdesenvolvidos ${ }^{4}$. Esses pacientes têm sido encaminhados, com frequência crescente, aos ambulatórios de gastropediatria e nutrição, demandando diagnósticos e condutas relacionadas ao seu estado nutricional. Para amparar tais decisões, o presente trabalho pretende avaliar os valores antropométricos e as variáveis relacionadas ao comprometimento nutricional de crianças com PC.

\section{M É T O D O S}

Estudo transversal incluiu 114 crianças com idades entre 2 a 12 anos, atendidas nos ambulatórios pediátricos de gastropediatria, ortopedia e neurologia infantil de um hospital terciário universitário. Os dados foram colhidos no período de junho de 2004 a janeiro de 2006. Foram incluídas crianças com o diagnóstico confirmado por neurologista, cujos pais concordaram e assinaram o Termo de Consentimento Livre e Esclarecido. Foram excluídas crianças com doenças metabólicas e síndromes genéticas.

Para cálculo do tamanho da amostra considerou-se proporção de população finita para estudo descritivo, priorizou-se a variável Escore-Z $\leq-2$ e utilizaram-se dados do projeto-piloto realizado em 40 pacientes. O tamanho da população-alvo era de 160 pacientes com diagnóstico de PC, habitualmente atendidos no hospital. A proporção de indivíduos com a característica de interesse, Escore-Z $\leq-2$, no projeto piloto, foi de $45 \%$. A diferença desejada entre proporção amostral e proporção populacional (erro amostral) foi de 0,05. Assim o cálculo da amostra foi de 113 crianças para esse estudo. 
Para aferição das medidas antropométricas foram utilizados as seguintes técnicas e instrumentos:

- Altura (direta): a criança foi medida em um antropômetro vertical fixo, descalça e com os pés juntos, em posição ereta e olhando à frente, com braços ao longo do corpo e o dorso, as nádegas e a cabeça encostados no plano vertical do antropômetro.

- Altura (estimada): a obtenção da altura indireta foi utilizada quando havia impossibilidade em estabelecer a posição ortostático, segundo método desenvolvido por Stevenson². Utilizou-se um paquímetro ósseo, em centímetros, para medir a distância da altura do joelho ao calcanhar, com um ângulo de $90^{\circ}$ entre a perna e a coxa do paciente.

- Peso: a criança foi pesada em uma balança eletrônica digital, com capacidade de $150 \mathrm{~kg}$, calibrada para zero, para obter peso em $\mathrm{kg}$. A criança estava sem sapatos e com roupas leves. Sempre que necessário, a criança foi pesada no colo do responsável, e posteriormente, seu peso foi calculado pela diferença das duas pesagens.

- Perímetro do Braço (PB): o braço da criança foi posicionado em atitude de flexão. Com uma fita métrica inelástica, o ponto médio entre o processo acrômial (ombro) da escápula e do olécrano (cotovelo) foi marcado com caneta hidrográfica. O perímetro do braço foi então mensurado com o membro pendente no ponto marcado.

- Prega Cutânea Tricipital (PCT): adipômetro Lange ${ }^{\circledast}$ foi aplicado suavemente no mesmo ponto utilizado para obtenção do PB e foi feita a medida da prega.

O Índice de Massa Corporal (IMC) foi calculado utilizando o peso e altura.

Os valores do National Center for Health and Statistics (NCHS) ${ }^{8}$ foram utilizados como referência de normalidade para comparação do peso, altura e IMC. Com o perímetro do braço e a prega cutânea tricipital, foram calculadas a Área Muscular do Braço (AMB) e a Área Gordurosa do Braço
(AGB); para esses indicadores a referência para comparação foi Frisancho ${ }^{9}$.

Os pacientes foram definidos como desnutridos quando o indicador antropométrico relacionado por idade, em Escore-Z, assumiu valor $\leq-2$.

As medidas antropométricas foram tomadas no lado direito da criança e considerada a média de três mensurações consecutivas.

A avaliação da classe social das famílias foi feita por método de pontuação, adaptado da ABEP10, no qual as famílias foram classificadas de maneira pertinente, dentro de uma das classes sociais $A, B, C, D$, e E.

A classificação do tipo de PC foi obtida do prontuário do paciente, conforme diagnóstico estabelecido pelo neurologista pediátrico que acompanhou o paciente no mesmo serviço de assistência médica.

As seguintes informações foram colhidas com o cuidador por meio de entrevista estruturada: número de internações, antecedente pessoal de pneumonia, habilidade para alimentar-se independentemente, dificuldade para deglutir (caracterizada como presença de sinais de aspiração, como: tosse, engasgo, respiração ruidosa ou cianose ao ingerir alimentos nas consistências líquida, pastosa ou sólida, de acordo com informação do cuidador e com frequência regular), orientação nutricional (ter recebido orientação nutricional, ministrada por nutricionista, independente do número de sessões).

As variáveis dependentes, medidas dos valores antropométricos, foram transformadas em Escore-Z e incluíram os seguintes indicadores: peso, altura, IMC, áreas muscular e gordurosa do braço, e foram relacionadas às seguintes variáveis independentes: idade, gênero, classe social, tipo de PC, número de internações, história de pneumonia, habilidade em alimentar-se independentemente, dificuldade em deglutir e orientação nutricional prévia.

Para registro e análise dos dados usou-se o programa Statistical Package for the Social 
Sciences ${ }^{11}$. Para comparação das médias empregou-se o teste $t$ de Student ou análise de variância e para análise multivariada, a regressão linear múltipla, método stepwise forward, adotando-se o valor de $p<0,05$ para inclusão de variáveis no modelo matemático. O nível de significância adotado foi de 5\%.

A pesquisa foi aprovada pelo Comitê de Ética em Pesquisa, Faculdade de Ciências Médicas. Universidade Estadual de Campinas (processo $n^{\circ} 283 / 2004$ ).

\section{RES ULTA D O S}

A pesquisa incluiu 114 crianças com idade entre 2 a 12,9 anos, sendo 65 (57,0\%) do sexo masculino. A classe social $D$ foi a mais frequente $(46,5 \%, n=53)$, seguida pela C (39,5\%, $n=45), B$ $(12,3 \%, n=14)$ e a $E(1,8 \%, n=2)$.O tipo dominante de PC foi o tetraparético $(68,4 \%, n=78)$, na sequência o misto $(13,2 \%, n=15)$, hemiparético $(8,8 \%, n=10)$, diparético $(7,0 \%, n=8)$ e atetóide $(2,6 \%, n=3)$. O tipo atáxico só foi encontrado na forma mista.

Cento e três crianças $(90,4 \%)$ tinham história de pelo menos uma internação. As crianças tetraparéticas apresentaram maior número de internações (103/114). História de pneumonia foi positiva em 67 crianças (58,8\%).

Dificuldade em deglutir foi referida por 66 cuidadores $(57,9 \%)$ e a referência de inabilidade para alimentar-se independentemente em 73 $(64,0 \%)$ crianças. Considerando o período anterior ao estudo, desde o nascimento da criança, apenas
15 (13,2\%) famílias tinham recebido orientação nutricional por nutricionista.

A maior frequência de desnutrição foi encontrada na relação peso/idade. Na sequência foram mais acometidas as medidas IMC, altura, $A G B$ e $A M B$, conforme descrição a seguir.

Cinquenta e oito pacientes (50,9\%) apresentaram valores inferiores a -2 no Escore- $Z$ de peso para idade. Para o Escore-Z de altura para idade, 44 pacientes $(38,6 \%)$ apresentaram valores inferiores ou iguais a -2 . Valores descritivos para esses indicadores são apresentados nas Tabelas 1 e 2 .

Os valores dos Escores-Z do índice de massa corporal foram inferiores ou iguais a $-2 \mathrm{em}$ 52 crianças $(45,6 \%)$. Os indivíduos com PC atetóide apresentaram média significantemente mais baixa. Na Tabela 3 são apresentados os valores de Escore-Z para IMC, AMB e AGB e respectivos valores de significância dos fatores relacionados ao estado nutricional.

Trinta e quatro pacientes (29,8\%) apresentaram valores de Escore-Z da área muscular do braço $\leq-2$. Quarenta pacientes $(35,1 \%)$ apresentaram valores de escore- $Z$ de área gordurosa do braço inferiores ou iguais a -2 . Os valores descritivos desses indicadores são apresentados na Tabela 3.

A análise multivariada, método stepwise forward pode ser observada na Tabela 4, onde são apresentados os fatores que influenciaram significativamente o estado nutricional das crianças com paralisia cerebral como: dificuldade para deglutir, relacionada com desnutrição identificada

Tabela 1. Valores descritivos dos indicadores antropométricos de 114 crianças com paralisia cerebral, apresentados em Escore-Z, referência NCHS. Campinas (SP), 2004-2006.

\begin{tabular}{lccccc}
\hline Indicares nutricionais & Média & Desvio-Padrão & Mínimo & Mediana & Máximo \\
\hline Peso & $-2,19$ & 2,48 & $-8,34$ & $-2,12$ & 2,90 \\
Altura & $-1,66$ & 1,50 & $-6,77$ & $-1,41$ & 1,55 \\
IMC & $-1,74$ & 2,75 & $-8,55$ & $-1,36$ & 2,76 \\
AMB & $-1,03$ & 1,73 & $-4,93$ & $-1,14$ & 4,66 \\
AGB & $-1,15$ & 1,41 & $-3,33$ & $-1,41$ & 4,67 \\
\hline
\end{tabular}

IMC: índice de massa corporal; AMB: área muscular do braço; AGB: área de gordura do braço; NCHS: National Center for Health and Statistics. 
Tabela 2. Fatores que interferiram no peso e na altura em relação à idade das 114 crianças com paralisia cerebral. Campinas (SP), 2004-2006.

\begin{tabular}{|c|c|c|c|c|c|}
\hline \multirow{3}{*}{ Fatores estudados } & \multirow{3}{*}{ Pacientes ( $\mathrm{n}$ ) } & \multicolumn{4}{|c|}{ Escore-Z } \\
\hline & & \multicolumn{2}{|c|}{ Peso } & \multicolumn{2}{|c|}{ Altura } \\
\hline & & Média & Desvio-Padrão & Média & Desvio-Padrão \\
\hline \multicolumn{6}{|c|}{ Tipo de paralisia cerebra $\|^{* *}$} \\
\hline Tetraparético & 78 & $-2,31$ & 2,36 & $-1,64$ & 1,36 \\
\hline Diparético & 8 & $-0,47$ & 2,00 & $-1,50$ & 0,90 \\
\hline Hemiparético & 10 & $-0,73$ & 3,57 & $-1,17$ & 2,97 \\
\hline Atetóide & 3 & $-3,55$ & 2,87 & $-2,14$ & 1,17 \\
\hline \multirow[t]{2}{*}{ Misto } & 15 & $-3,21$ & 1,62 & $-2,10$ & 1,08 \\
\hline & & \multicolumn{2}{|c|}{$p=0,024$} & \multicolumn{2}{|c|}{$p=0,004$} \\
\hline \multicolumn{6}{|l|}{ Classe social ${ }^{\star *}$} \\
\hline B & 14 & $-3,09$ & 3,00 & $-1,81$ & 1,43 \\
\hline C & 45 & $-1,59$ & 2,33 & $-1,43$ & 1,45 \\
\hline D & 53 & $-2,57$ & 2,35 & $-1,88$ & 1,52 \\
\hline \multirow[t]{2}{*}{ E } & 2 & 0,44 & 1,19 & $-0,08$ & 1,53 \\
\hline & & \multicolumn{2}{|c|}{$p=0,045$} & \multicolumn{2}{|c|}{$p=0,284$} \\
\hline \multicolumn{6}{|c|}{ Alimentação independente ${ }^{* * *}$} \\
\hline $\operatorname{sim}$ & 41 & $-0,74$ & 2,10 & $-1,39$ & 1,61 \\
\hline \multirow{2}{*}{ Não } & 73 & $-3,01$ & 2,30 & $-1,82$ & 1,42 \\
\hline & & \multicolumn{2}{|c|}{$p=0,001$} & \multicolumn{2}{|c|}{$p=0,06$} \\
\hline \multicolumn{6}{|c|}{ Dificuldade em deglutir líquido*** } \\
\hline $\operatorname{sim}$ & 37 & $-3,68$ & 2,34 & $-2,15$ & 1,42 \\
\hline \multirow[t]{2}{*}{ Não } & 77 & $-1,48$ & 2,22 & $-1,43$ & 1,48 \\
\hline & & \multicolumn{2}{|c|}{$p=0,001$} & \multicolumn{2}{|c|}{$p=0,012$} \\
\hline \multicolumn{6}{|c|}{ Dificuldade em deglutir sólido ${ }^{* * *}$} \\
\hline $\operatorname{sim}$ & 63 & $-3,30$ & 2,23 & $-1,98$ & 1,41 \\
\hline \multirow[t]{2}{*}{ Não } & 51 & $-0,83$ & 2,06 & $-1,27$ & 1,53 \\
\hline & & \multicolumn{2}{|c|}{$p=0,001$} & \multicolumn{2}{|c|}{$p=0,004$} \\
\hline
\end{tabular}

Houve diferença significante $p<0,05\left({ }^{* *}\right.$ Análise de variância ou ${ }^{* * *}$ teste $t$ Student).

em todos os indicadores antropométricos estudados, inabilidade em comer independentemente e história de pneumonia para o IMC, classe social para $A M B$ e idade para $A G B$.

\section{I S C U S S Ã O}

Os fatores associados ao potencial biológico de crescimento das crianças com sequelas de injúria cerebral e os padrões de crescimento determinados por esse potencial são incompletamente conhecidos ${ }^{2}$. As razões do crescimento limitado nas crianças com PC provavelmente são multifatoriais e podem incluir fatores nutricionais, hormonais, osteomusculares e neurológicos ${ }^{2,12}$.
O conjunto dos valores antropométricos, na amostra estudada, pode ser resumido na identificação de altas porcentagens de crianças apresentando comprometimento nutricional, em diferentes indicadores, padrão similar ao encontrado em estudos internacionais ${ }^{3,12-14}$.

Mais da metade das crianças apresentou média de Escore-Z da medida peso/idade $\leq-2$. Em estudos internacionais que mensuraram os indicadores peso/idade e altura/idade, os valores encontrados foram reportados de diferentes maneiras. Stevenson ${ }^{5}$ encontrou $19 \%$ das crianças com peso/altura abaixo do percentil 50, Krick et al. ${ }^{12}$ reportaram que $10 \%$ das crianças, na idade de 8 anos, apresentavam altura/idade inferior à 
Tabela 3. Fatores que interferiram no índice de massa corporal, na área muscular e de gordura do braço em relação à idade das 114 crianças com paralisia cerebral. Campinas (SP), 2004-2006.

\begin{tabular}{|c|c|c|c|c|c|c|c|}
\hline \multirow{2}{*}{$\begin{array}{l}\text { Escores Z } \\
\text { Fatores estudados }\end{array}$} & \multirow[b]{2}{*}{ Pacientes } & \multicolumn{2}{|c|}{ IMC } & \multicolumn{2}{|c|}{ AMB } & \multicolumn{2}{|c|}{$A G B$} \\
\hline & & Média & DP & Média & DP & Média & DP \\
\hline \multicolumn{8}{|c|}{ Tipo de paralisia cerebral ${ }^{*}$} \\
\hline Tetraparético & 78 & $-2,07$ & 2,87 & $-1,16$ & 1,63 & $-1,30$ & 1,27 \\
\hline Diparético & 8 & 0,33 & 2,12 & 0,32 & 2,16 & 0,22 & 1,15 \\
\hline Hemiparético & 10 & 0,35 & 2,13 & $-0,65$ & 2,32 & 0,17 & 2,01 \\
\hline Atetóide & 3 & $-2,83$ & 2,70 & $-1,72$ & 0,28 & $-2,08$ & 1,15 \\
\hline \multirow[t]{2}{*}{ Misto } & 15 & $-2,30$ & 1,68 & $-1,17$ & 1,53 & $-1,82$ & 0,81 \\
\hline & & \multicolumn{2}{|c|}{$p=0,005$} & \multicolumn{2}{|c|}{$p=0,169$} & \multicolumn{2}{|c|}{$p<0,001$} \\
\hline \multicolumn{8}{|l|}{ Classe social $^{\star *}$} \\
\hline B & 14 & $-2,58$ & 3,41 & $-1,76$ & 1,83 & $-1,44$ & 1,36 \\
\hline C & 45 & $-1,36$ & 2,76 & $-1,03$ & 1,77 & $-0,83$ & 1,66 \\
\hline$D$ & 53 & $-1,91$ & 2,55 & $-0,94$ & 1,39 & $-1,37$ & 1,16 \\
\hline \multirow[t]{2}{*}{$E$} & 2 & 0,06 & 2,75 & 1,71 & 4,16 & $-0,63$ & 1,70 \\
\hline & & \multicolumn{2}{|c|}{$p=0,373$} & \multicolumn{2}{|c|}{$p=0,052$} & \multicolumn{2}{|c|}{$p=0,266$} \\
\hline \multicolumn{8}{|c|}{ Alimentação independente ${ }^{* * *}$} \\
\hline Sim & 41 & 0,01 & 2,01 & $-0,09$ & 1,84 & $-0,31$ & 1,56 \\
\hline \multirow[t]{2}{*}{ Não } & 73 & $-2,72$ & 2,63 & $-1,56$ & 1,42 & $-1,62$ & 1,07 \\
\hline & & \multicolumn{2}{|c|}{$p<0,001$} & \multicolumn{2}{|c|}{$p<0,001$} & \multicolumn{2}{|c|}{$p<0,001$} \\
\hline \multicolumn{8}{|c|}{ Dificuldade em deglutir líquido*** } \\
\hline Sim & 37 & $-3,37$ & 2,86 & $-1,88$ & 1,51 & $-1,82$ & 0,98 \\
\hline \multirow[t]{2}{*}{ Não } & 77 & $-0,95$ & 2,34 & $-0,62$ & 1,69 & $-0,83$ & 1,48 \\
\hline & & \multicolumn{2}{|c|}{$p<0,001$} & \multicolumn{2}{|c|}{$p<0,001$} & \multicolumn{2}{|c|}{$p<0,001$} \\
\hline \multicolumn{8}{|c|}{ Dificuldade em deglutir sólido*** } \\
\hline $\operatorname{Sim}$ & 63 & $-2,98$ & 2,54 & $-1,71$ & 1,39 & $-1,82$ & 0,91 \\
\hline \multirow[t]{2}{*}{ Não } & 51 & $-0,21$ & 2,20 & $-0,19$ & 1,74 & $-0,33$ & 1,50 \\
\hline & & \multicolumn{2}{|c|}{$p<0,001$} & \multicolumn{2}{|c|}{$p<0,001$} & \multicolumn{2}{|c|}{$p<0,001$} \\
\hline
\end{tabular}

DP: Desvio-padrão; N: número de pacientes; IMC: índice de massa corporal; AMB: área muscular do braço; AGB: área de gordura do braço. Houve diferença significante $p<0,05\left({ }^{* *}\right.$ Análise de variância ou ${ }^{* * *}$ teste $t$ Student).

Tabela 4. Análise multivariada, método stepwise, das medidas antropométricas por idade em valores de Escore-Z. Campinas (SP), 2004-2006.

\begin{tabular}{lcc}
\hline Variáveis (Escore-Z) & $\mathrm{R}_{2}$ ajustado $(\%)$ & Modelo \\
\hline Peso $^{1}$ & 27,2 & $\mathrm{Y}=-0,758-1,915 \times \mathrm{DDS}-1,164 \times \mathrm{DDL}$ \\
Altura $^{2}$ & 13,1 & $\mathrm{Y}=-1,640+1,728 \times \mathrm{PCH}-1,022 \times \mathrm{DDP}$ \\
$\mathrm{IMC}^{3}$ & 31,9 & $\mathrm{Y}=31,333-8,074 \times \mathrm{DDS}-7,3 \times \mathrm{Al}+4,37 \times \mathrm{P}$ \\
$\mathrm{AMB}^{4}$ & 22,4 & $\mathrm{Y}=-0,240-1,518 \times \mathrm{DDL}+2,714 \times \mathrm{CSE}$ \\
$\mathrm{AGB}^{5}$ & 31,8 & $\mathrm{Y}=0,013+0,038 \times \mathrm{DDS}-0,002 \times \mathrm{Idade}$ \\
\hline
\end{tabular}

IMC: índice de massa corporal; AMB: área muscular do braço; AGB: área de gordura do braço; DDS: dificuldade em deglutir sólido; DDL: dificuldade em deglutir líquido; PCH: paralisia cerebral hemiparética; DDP: dificuldade em deglutir pastoso; Al: alimentar-se independente; $\mathrm{P}$ : presença de pneumonia; CSE: classe social $\mathrm{E}_{;} \mathrm{R}_{2}$ : coeficiente de determinação.

$\mathrm{Y}=$ modelo matemático, onde: ${ }^{1} \mathrm{Y}=\mathrm{Z}$ peso; ${ }^{2} \mathrm{Y}=\mathrm{Z}$ altura (não incluídos os dois casos $\left.\mathrm{Z}<-5,5\right) ;{ }^{3} \mathrm{Y}=(Z \mathrm{IMC}){ }^{1,5}+10 ;{ }^{4} \mathrm{Y}=\mathrm{Z} \mathrm{AMB} ;{ }^{5} \mathrm{Y}=1 /(\mathrm{Z}$ $A G B+10)^{2}$.

referência NCHS, Samson-Fang \& Stevenson ${ }^{14}$, identificaram valores da relação peso/altura inferiores ao percentil $10 \mathrm{em} 45 \%$ das crianças, e Sullivan et al. ${ }^{15}$ identificaram comprometimento nutricional, para essa medida, em 38\% da casuística e, ainda, nos pacientes com comprometimento motor intenso, a desnutrição atingiu frequência de $49 \%$. 
Nos países em desenvolvimento, ao lado dos determinantes biológicos, devem ser considerados os fatores socioeconômicos que, muitas vezes, atuam prejudicando o crescimento de todas as crianças e, em particular, daquelas com necessidades específicas. Nesta pesquisa, a maioria das crianças pertencia à classe social $D$, na qual fatores ambientais associados a dificuldades sócioeconômicas atuam de maneira desfavorável no crescimento de crianças $^{16}$. As crianças com PC do tipo tetraparético são caracterizadas por comprometimento motor grave, alta frequência de distúrbio de deglutição e maior número de ocorrências mórbidas ${ }^{6,17}$. Esse tipo de PC foi o mais frequente na amostra estudada e, certamente, influenciou os resultados obtidos.

Muitas crianças avaliadas no presente estudo poderiam ter sido beneficiadas por investigação e manejo nutricional como parte de seus cuidados integrais desde idades precoces, porém poucas crianças receberam orientação nutricional ou qualquer recomendação nutricional especializada até o momento da presente avaliação. Muitas vezes, o pediatra é o primeiro profissional a entrar em contato com esses pacientes. Assim, é este profissional quem deve indicar, o mais oportunamente possível, a necessidade de acompanhamento multidisciplinar ${ }^{18}$.

Estudos internacionais relataram dados referentes ao acompanhamento longitudinal do estado nutricional de crianças com PC, obtidos antes e após a realização de gastrostomia como via alimentar alternativa ${ }^{19-22}$. A gastrostomia está associada com ganho de peso, diminuição das ocorrências de doenças pulmonares e das taxas de mortalidade ${ }^{23-25}$. É evidente a necessidade de identificar e conduzir distúrbios da deglutição, mesmo que, em muitos pacientes, o tratamento possa implicar administração dos alimentos por vias alternativas da via oral. Tais estudos, no entanto, não avaliaram a melhor oportunidade para início dessas intervenções, uma informação necessária para reprodução dos bons resultados em populações seguidas por gastroenterologistas, pediatras e nutricionistas. Estudos que identifi- quem o momento mais adequado para instalação de vias alimentares alternativas são necessários.

A associação de distúrbio da deglutição e prejuízo nutricional tem sido comprovada em pacientes neuropatas. Hung et al. ${ }^{26}$ avaliaram 75 crianças com paralisia espástica e idade variando de 5 meses a 10 anos. Na análise multivariada, a desnutrição associou-se significantemente ao gênero feminino, distúrbio da deglutição, refeição com longa duração e limites na comunicação verbal ${ }^{26}$. Vários estudos verificaram que a inabilidade em alimentar-se independentemente teve relação significativa com a desnutrição nas crianças com $P C^{6,17,27-29}$. Na presente pesquisa, a dificuldade em deglutir foi identificada em todos os tipos de PC, com exceção do diparético. Esse distúrbio associou-se à desnutrição para todas as relações antropométricas. O pediatra deveria estar atento para o diagnóstico precoce de dificuldades de deglutição em lactentes com diagnóstico ou histórico de fatores de risco para PC.

Os presentes resultados, provenientes de um estudo transversal, não podem ser generalizados ou servir como referência para a população brasileira. Contudo, considerando os dados epidemiológicos disponíveis ${ }^{4}$, será cada vez mais frequente a necessidade de identificar o estado nutricional de crianças com paralisia cerebral e de decidir as necessidades terapêuticas nessas crianças. Um desenho longitudinal conduzido em pacientes contemplados com atendimento multidisciplinar e iniciado precocemente na vida desses pacientes poderá ser mais adequado para estabelecer valores de referência. A metodologia utilizada na presente pesquisa para aquisição de dados antropométricos foi identificada como um método que pode ser aplicado com rapidez e baixo custo em crianças com PC.

\section{CONCLUSÃO}

A amostra de crianças com paralisia cerebral caracterizou-se por alta frequência de desnutrição. Peso, altura, IMC e avaliação de massa gordurosa e muscular mostraram valores signifi- 
cantemente inferiores aos das tabelas de referência do NCHS. Escores-Z $\leq-2$ para as relações peso/idade e IMC/idade foram observados em respectivamente $50,8 \%$ e $45,5 \%$ das crianças. Um terço das crianças apresentou desnutrição em outros parâmetros antropométricos, incluindo altura, prega cutânea tricipital, massa muscular e gordurosa do braço. O tipo tetraparético de PC foi o que apresentou maiores frequência e gravidade de comprometimento nutricional, com $72,4 \%, 73,1 \%$ e $75,6 \%$ de desnutridos respectivamente para peso, IMC e área gordurosa do braço.

A dificuldade em deglutir foi o fator que influenciou significativamente o estado nutricional das crianças, interferindo negativa e globalmente em todos os indicadores.

\section{COLABORADORES}

A.L.A. CARAM desenvolveu a pesquisa, coletou os dados e redigiu o artigo A.M. MORCILLO co-orientou o projeto, fez a análise estatística e participou da redação do artigo. E.A.L.C. PINTO orientou o projeto como um todo e participou da redação do artigo.

\section{REFERÊ N CIAS}

1. Fajardo AP, Berger C, Ramos DD, Cunha J, Livi KL, Lenz MLM. A atenção à saúde da criança de zero a cinco anos de idade: protocolo. Porto Alegre: Secretaria Municipal da Saúde; 2004 [Internet]. [acesso 2008 maio 15]. Disponível em: <http// www2.ghc.com.br/GepNet/protocolopmpoa. $\mathrm{pdf}>$.

2. Stevenson RD. Beyond growth: gastrostomy feeding in children with cerebral palsy. Develop Med Child Neurol. 2005; 47(2):76. doi: 10.1017/s00 12162205000150.

3. Samson-Fang $L$, Fung $E$, Satallings VA, Conaway M, Worley G, Rosenbaum P, et al. Relationship of nutritional status to health and societal participation in children with cerebral palsy. J Pediatr. 2002; 141(5):637-43. doi: 10.1067/mpd. 2002.12988.

4. Piovesana AMSG, Val Filho JAC, Lima CLA, Fonseca MS, Mürer AP. Encefalopatia crônica: paralisia cerebral. In: Fonseca LF, Pianetti G, Xavier CC.
Compêndio de neurologia infantil. Rio de Janeiro: Medsi; 2002. p.826-37.

5. Stevenson RD. Use of segmental measures to estimate stature in children with cerebral palsy. Arch Pediatr Adolesc Med. 1995; 149(6):658-62.

6 Sullivan PB, Juszczak E, Bachet AME, Thomas Ag, Lamert B, Vernon-Roberts A, et al. Impact of gastrostomy tube feeding on the quality of life of carers of children with cerebral palsy. Develop Med Child Neurol. 2004; 46(12):796-800. doi: 10.10 17/S0012162204001392.

7. Fung EB, Samson-Fang L, Stallings VA, Conaway M, Liptak G, Henderson RC. Feeding dysfunction is associated with poor and health status in children with cerebral palsy. J Am Diet Assoc. 2002; 102(3):361-73.

8. National Center for Health Statistics. CDC crowth charts. United States; 2000 [Internet]. [cited 2004 Feb]. Available from: <http://www.cdc.gov/growth charts/>.

9. Frisancho AR. Anthropometric standards for the assessment of growth and nutrition status. Ann Arbor: University of Michigan Press; 1993.

10. Associação Brasileira de Empresas de Pesquisas. Critério de classificação econômica Brasil 2003. Disponível em: <www.abep.org>.

11. Statistical Package for Social Sciences. SPSS for Windows, Rel. 11.0.1. 2001. versão 11.0. Chicago: SPSS; 2001.

12. Krick J, Murphy-Miller P, Zeger S, Weight E. Pattern of growth in children with cerebral palsy. J Am Diet Assoc. 1996; 96(7):680-5.

13. Silva Netto CR. Deglutição na criança, no adulto e no idoso: fundamentos para odontologia e fonoaudiologia. São Paulo: Lavise; 2003. p.176.

14. Samson-Fang LJ, Stevenson RD. Identification in children with cerebral palsy: poor performance of weight-for height centiles. Develop Med Child Neurol. 2000; 42(3): 162-8. doi:10.1017/S00121 62200000293.

15. Sullivan PB, Juszczak E, Lamert B, Rose M, FordAdams ME, Johnson A. Impact of feeding problems on nutrition intake and growth: Oxford Feeding Study II. Develop Med Child Neurol. 2002, 44(7): 461-7. doi: 10.1017/s0012162204001392.

16. Gallo PR, Leone C, Amigo H. Fatores de risco ao retardo de crescimento estatural em crianças de baixo nível econômico e social de São Paulo, Brasil. Arch Latinoam Nutr. 2000, 50(2):121-6.

17. Gangil A, Patwari AK, Aneja S, Ahuja B, Anand VK. Feeding problems in children with cerebral palsy. Ind Pediatr. 2001; 38(6):839-46. 
18. Rotta NT. Paralisia cerebral, novas perspectivas terapêuticas. J Pediatr (Rio de Janeiro). 2002; 78 (Supl.1):S48-S54.

19. Sleigh G, Brocklehurst P. Gastrostomy feeding in cerebral palsy: a systematic review. Arch Dis Child. 2004, 89(6):534-9. doi: 10.1136/adc.2002.0211 70.

20. Issacs D, Kilham HA, Somervile HM, Oloughlin EV, Tobin B. Nutrition in cerebral palsy. J Paediatr Child Health. 2004; 40(5-6):308-10.

21. Rogers B. Feeding method and health outcomes of children with cerebral palsy. J Pediatr. 2004; 145(2):S28-S32.

22. Sullivan PB, Juszczak E, Bachet AME, Lamert B, Vernon-Roberts A, Grant HW, et al. Gastrostomy tube feeding in children with cerebral palsy: a prospective, longitudinal study. Develop Med Child Neurol. 2005; 47(2):77-85. doi: 10.1017/S0012 162205000162.

23. Strauss D, Shavelle R, Reynolds R, Rosenbloom L, Day S. Survivel in cerebral palsy in the last 20 years: signs of improvement? Dev Med Child Neurol. 2007; 49(2):86-92.

24. Sullivan PB, Morrice JS, Vernon-Roberts A, Grant $\mathrm{H}$, Eltumi M, Thomas AG. Does gastrostomy tube feeding in children with cerebral palsy increase the risk of respiratory morbidity? Arch Dis Child.
2006; 91(6):458-60. doi: 10.1136/adc.2005.084 442.

25. Sullivan PB, Alder N, Bachlet AM, Grant H, Juszczak E, Henry J, et al. Gastrostomy feeding in cerebral palsy: too much of a good thing? Dev Med Child Neurol. 2006; 48(11):869. doi: 10.1017/S00121 62206001927.

26. Hung JW, Hsu TJ, Wu PC, Leong CP. Risk factors of undernutrition in children with spastic cerebral palsy. Chang Gung Med J. 2003; 26(6):425-32.

27. Tawfik R, Dickson A, Clarke M, Thomas AG. Caregives' perceptions following gastrostomy in severely disabled children with feeding problems. Develop Med Child Neurol. 1997; 39(11):746-51.

28. Thomas AG, Akobeng AK. Technical aspects of feeding the disabled child. Cur Opinion Clin Nutr Metab Care. 2000; 3(5):213-5.

29. Henderson RC, Grossberg RI, Matuszewski J, Menon N, Johnson J, Kecskemethy $\mathrm{HH}$, et al. Growth and nutritional status in residential center versus home-living children and adolescents with quadriplegic cerebral palsy. J Pediatr. 2007; 151(2): 161-6.

Recebido em: 11/6/2008

Versão final reapresentada em: 13/10/2009 Aprovado em: 11/11/2009 